\title{
Cognitive Slowing in Parkinson's Disease: A Behavioral Evaluation Independent of Motor Slowing
}

\author{
Nobukatsu Sawamoto, ${ }^{1}$ Manabu Honda, ${ }^{1,2,3}$ Takashi Hanakawa, ${ }^{1}$ Hidenao Fukuyama, ${ }^{1}$ and \\ Hiroshi Shibasaki, ${ }^{1}$ \\ ${ }^{1}$ Department of Brain Pathophysiology, Human Brain Research Center, Kyoto University Graduate School of Medicine, \\ Kyoto 606-8507, Japan, ²Laboratory of Cerebral Integration, National Institute for Physiological Sciences, Okazaki \\ 444-8585, Japan, and ${ }^{3} P R E S T O$, Japan Science and Technology Corporation, Kawaguchi 332-0012, Japan
}

Parkinson's disease (PD) is attributable primarily to depletion of dopamine in the basal ganglia, but the full effects of this depletion are unknown. It is well known that PD involves motor slowing, and although it is not easy to distinguish between the motor and cognitive components of behavior, clinical observations suggest that cognitive processing may also be compromised. However, it remains unclear whether such cognitive involvement exists, and if so, to what extent. Previous studies of cognitive slowing in PD have yielded conflicting results. This may be attributable to variations in experimental procedures, because most of the experiments used reaction-time tasks, which are inevitably confounded by motor components. In the present study, we evaluated the speed of cognitive processing in patients with PD without bradykinesia as a variable. We developed a mental-operation task that required serial updating of mental representations in response to a series of visual stimuli. By changing the speed of visual presentation and evaluating performance accuracy, the speed of cognitive processing was assessed independently of motor slowing. Cognitive impairment in PD became evident when higher speeds of cognitive processing (verbal more so than spatial) were required. In addition, cognitive slowing and motor slowing were significantly correlated. The results of the present study suggest that slowing in PD is not restricted to the motor domain but can be generally observed in other domains of behavior, including cognitive mental operations.

Key words: Parkinson's disease; mental-operation task; reaction-time task; cognitive slowing; bradykinesia; cognitivemotor interaction
Slowing of movement, or bradykinesia, is one of the characteristics of Parkinson's disease (PD). The slowing appears to be attributable primarily to a deficit in cognitive motor control (i.e., an ability to arrange complex movement), rather than simply an impairment in motor initiation and execution (Marsden, 1982). Slowing in imagery of movements and mental rotation of objects has also been suggested to occur in PD (Dominey et al., 1995; Lee et al., 1998). Although it is not easy to distinguish between the motor and cognitive components of behavior, clinical observations indicate that slowing in PD is not restricted to motor functioning but also involves cognitive functioning (Naville, 1922; Rogers, 1986).

The presence of cognitive slowing has been tested not only to clarify this aspect of PD itself but also to understand the function of the basal ganglia. Striatal dysfunction is coupled with motor slowing in PD (Vingerhoets et al., 1997). Although a role of the

Received Oct. 22, 2001; revised March 21, 2002; accepted March 29, 2002.

This work was supported in part by a Grant-in-Aid for Scientific Research for Future Program JSPS-RFTF97L00201 from the Japan Society for the Promotion of Science; by Priority Areas (C) Advanced Brain Science 12210012 from the Japan Ministry of Education, Science, Sports, and Culture to H.S.; by Special Coordination Funds for Promoting Science and Technology and a Grant-in-Aid for Scientific Research on Priority Areas (C) Advanced Brain Science 13210143 to M.H.; and by a Grant-in-Aid from the Japan Society for the Promotion of Science Research Fellows to N.S. N.S. is supported by Research Fellowships of the Japan Society for the Promotion of Science for Young Scientists. We thank Drs. J. Oita (Hikone City Hospital, Hikone, Japan), N. Kohara (Kobe City General Hospital, Kobe, Japan), M. Kanda, K. Terada, and A. Ohtsuka (Takeda General Hospital, Kyoto, Japan) for their support. We also thank Dr. T. Hamada (Kyoto University, Kyoto, Japan) for statistical advice and Dr. J. Kahle for skillful editing.

Correspondence should be addressed to Dr. Manabu Honda, Laboratory of Cerebral Integration, National Institute for Physiological Sciences, 38 Nishigonaka, Myodaiji, Okazaki 444-8585, Japan. E-mail: honda@nips.ac.jp.

Copyright (ㄷ) 2002 Society for Neuroscience $\quad 0270-6474 / 02 / 225198-06 \$ 15.00 / 0$ basal ganglia has been demonstrated in nonmotor cognitive operations as well as in motor control (Middleton and Strick, 1994), previous studies have produced conflicting results on the presence of cognitive slowing in PD (Rafal et al., 1984; Ransmayr et al., 1990; Poewe et al., 1991; Revonsuo et al., 1993; Berry et al., 1999). The results of some studies support the presence of slowing (Wilson et al., 1980; Pillon et al., 1989; Cooper et al., 1994; Pate and Margolin, 1994; Lee et al., 1998), whereas the results of others do not (Lafleche et al., 1990; Duncombe et al., 1994; Howard et al., 1994). This conflict may arise, at least in part, from differences in the experimental procedure used. To measure cognitive speed, most of the studies followed a reaction-time paradigm that used an identical motor response after a simple or complex cognitive task. This method carries the assumption that a difference in reaction time between the two tasks would correspond to the time required for cognitive processing, because the time required for the motor response is identical. However, this is debatable, because cognitive and motor processing may interact (Georgopoulos, 2000). In fact, previous studies of patients with PD suggest that there are impairments in the cognitive-motor interaction specifically (Schwab et al., 1954; Benecke et al., 1986, 1987; Agostino et al., 1992; Georgiou et al., 1993; Majsak et al., 1998).

The aim of the present study is to examine the speed of cognitive processing in patients with $\mathrm{PD}$ without motor response rate as a variable. For this purpose, we developed a mentaloperation task that required serial updating of a mental representation in response to a series of visual stimuli without involving any overt movement (Honda et al., 1998). The speed of visual presentation was systematically changed, and the accuracy of performance was evaluated as a function of the presentation 
Table 1. Clinical profiles and intelligence scales of patients and normal subjects

\begin{tabular}{lllllllll} 
Group & $n$ & Age & $\begin{array}{l}\text { Sex } \\
(\text { male:female) }\end{array}$ & $\begin{array}{l}\text { Education } \\
\text { (years) }\end{array}$ & MMSE & IQ & VAIS-R \\
\hline PD & 23 & $63.6 \pm 6.0$ & $13: 10$ & $11.7 \pm 2.9$ & $28.6 \pm 1.6$ & $102.8 \pm 8.6$ & $104.3 \pm 10.8$ & $100.1 \pm 9.0$ \\
Controls & 25 & $63.5 \pm 5.3$ & $12: 13$ & $11.5 \pm 2.5$ & $28.8 \pm 1.7$ & $102.4 \pm 8.0$ & $101.5 \pm 8.9$ & $102.5 \pm 9.5$ \\
\hline
\end{tabular}

Mean \pm SD. IQ, Total IQ; VIQ, verbal IQ; PIQ, performance IQ.

Table 2. Disease severity of PD patients $(n=23)$

\begin{tabular}{llll}
$\begin{array}{l}\text { Duration } \\
\text { (years })\end{array}$ & $\begin{array}{l}\text { L-dopa daily dose } \\
(\mathrm{mg})^{a}\end{array}$ & H\&Y & $\begin{array}{l}\text { UPDRS } \\
\text { total score }\end{array}$ \\
\hline $3.5 \pm 2.2(1-8)$ & $296 \pm 150(0-600)$ & $2.1 \pm 0.7(1-3)$ & $23.4 \pm 8.5(8-41)$
\end{tabular}

Mean \pm SD (range).

${ }^{a} \mathrm{~L}$-dopa was prescribed in combination with peripheral decarboxylase inhibitors.

speed. This approach enabled us to evaluate the speed of cognitive processing in patients with PD without motor slowing as a confounding factor.

\section{MATERIALS AND METHODS}

Subjects. Twenty-three mildly impaired patients with PD and 25 age-, education-, and sex-matched, healthy volunteers (controls) participated in the present study (Table 1). All of the subjects were younger than 75 years, and none of them had a history of treatment for neurological or psychiatric disorders other than PD. The patients were evaluated for parkinsonian symptoms using both the Hoehn and Yahr rating scale (H\&Y) and the Unified Parkinson's Disease Rating Scale (UPDRS) (Table 2). The patients continued to take medications such as L-3,4dihydroxyphenylalanine (L-DOPA), peripheral decarboxylase inhibitors, dopamine agonists, or anticholinergics, or any combination of these drugs, at the time of testing. Nevertheless, characteristic parkinsonian motor symptoms were still apparent. All of the patients were rated $\leq 3$ on the $\mathrm{H} \& \mathrm{Y}$ and scored $\leq 41$ on the UPDRS, indicating that the disability of all patients was relatively mild. The subjects were also screened for dementia using the Mini-Mental State Examination (MMSE) and the Japanese version of the Wechsler Adult Intelligence Scale-Revised (WAIS-R). The MMSE scores confirmed that participants were free of symptoms of dementia. All subjects who participated in the present study scored $>90$ on the WAIS-R. The PD and control groups were matched for MMSE and WAIS-R scores. All subjects gave written informed consent after a full explanation of the procedure.

Mental-operation tasks. We developed spatial (MO-s) and verbal (MO-v) versions of a mental-operation task. For both versions, a trial started with the presentation of a prime stimulus followed by a serial presentation of seven instruction stimuli. Visual stimuli were presented at the center of a computer monitor placed $\sim 50 \mathrm{~cm}$ from the subjects. Each stimulus subtended a $5.7^{\circ}$ visual angle, so that the subjects could easily recognize each stimulus at the center of view without moving their eyes. This minimized any influence of differences in oculomotor function between patients with PD and controls. During each trial, the subjects were asked to fixate on the visual stimuli and to avoid eye movement. Because the visual stimuli were always presented in the center of view, possible oculomotor dysfunction in patients with PD would be negligible.

For MO-s (Fig. 1A), the prime stimulus was a marker presented in one square of a grid subdivided into nine smaller squares; each instruction stimulus was either an arrow or a pair of tandem arrows pointing in one of four directions (up, down, right, or left). Subjects were requested to move the marker mentally from its starting square according to the instruction stimuli, on the grid that was no longer visible on the screen. In the example shown in Figure $1 A$, the subjects mentally moved the marker to the right by two squares, following double rightward arrows, and then downward by one square, following the next single downward arrow, and so forth. After each trial, the subjects reported the final location of the marker by pointing with their finger to one of the squares in the grid displayed on the monitor.

For MO-v (Fig. $1 B$ ), the prime stimulus was a kanji character indicating a day of the week, and each instruction stimulus was a randomly selected number from 1 to 3 . Subjects were instructed to advance the day of the week mentally in a serial manner, according to the instruction stimuli. In the example shown in Figure $1 B$, the subjects advanced the day from Friday to Saturday in response to the number 1, and from Saturday to Tuesday in response to the number 3. Although it is possible to add up all seven numbers and to advance the day by the remainder of division of the sum by 7 , subjects were explicitly asked to avoid this particular strategy. Special caution was given not to move the mouth or tongue during the session. After completing each trial, the day of the week they finally reached was verbally reported.

To test cognitive speed, the instructional stimulus frequency was systematically changed across the trials. Eight different stimulus frequencies (from 0.4 to $1.8 \mathrm{~Hz}$ in $0.2 \mathrm{~Hz}$ steps) were used for each of the two tasks. The perception of visual stimuli itself has been shown to be primarily intact in $\mathrm{PD}$, at least within the range of the stimulus presentation rate used in this study (Revonsuo et al., 1993). Ten trials were tested for each stimulus frequency, and the accuracy of the answer was evaluated for each trial. If the subjects were not able to follow the speed of visual presentation of the instruction stimuli, this was reflected in a lower performance accuracy. Thus, the speed of cognitive processing was evaluated without measuring reaction time.

After full instruction about the procedures, the MO-s and MO-v were tested. The order of the two tasks tested was balanced across the subjects in each group. Each task consisted of six blocks (Fig. 1C). Each block started with a trial at $0.4 \mathrm{~Hz}$. The stimulus frequency was increased to 1.8 $\mathrm{Hz}$ in $0.2 \mathrm{~Hz}$ steps and then decreased. Thus, one block consisted of two trials for each of the eight different stimulus frequencies. The first block was considered a practice block; it was excluded from the analysis. Therefore, a total of 80 trials (eight frequencies $\times$ two trials $\times$ five blocks) were analyzed for each task. The procedure lasted $\sim 50 \mathrm{~min}$.

Data analysis. All statistical analyses were performed using the Statistical Program for the Social Sciences (SPSS, Chicago, IL) on a microcomputer.

First, the number of correct answers (i.e., performance accuracy) was subject to repeated measures (RM)-ANOVA with stimulus frequency (eight speeds) and task (MO-s and MO-v) as within-subjects factors, and group (PD and controls) as a between-subjects factor. Unpaired Student's $t$ tests were also conducted to compare the performance between patients with PD and controls at each frequency for each task separately. In addition, to explore the difference in performance between the two tasks, separate RM-ANOVAs were also conducted for each group (within-subject factor of frequency and task). Differences with a value of $p<0.05$ after Greenhouse-Geisser correction were considered statistically significant.

The relationship between cognitive slowing and motor impairment in the patients with PD was examined. As an index of cognitive slowing, a "bradyphrenia score" was calculated as the number of wrong answers at $1.0 \mathrm{~Hz}$ for each of the tasks. This frequency was selected because the difference of performance between patients with PD and controls was most prominent at this frequency for both tasks (Fig. 2C). As an index of motor impairment, the bradykinesia subscale proposed in a previous study was used (Vingerhoets et al., 1997). This subscale was defined as the sum of the four subscales from the UPDRS motor examination section: speech, facial expressions, generalized bradykinesia, and six different types of repetitive limb moments. Although the bradykinesia subscale was not a direct measurement of motor slowing, it has been reported to correlate well with the Purdue pegboard test, which has been shown to be an objective measure of bradykinesia or motor slowing (Tiffin and Asher, 1948; Vingerhoets et al., 1997). Moreover, a positron emission tomography (PET) study demonstrated a strong relationship between the bradykinesia subscale and the degree of nigrostriatal dopaminergic deficit (Vingerhoets et al., 1997). Pearson's correlation coefficient between the two scores was calculated for each task. 
A

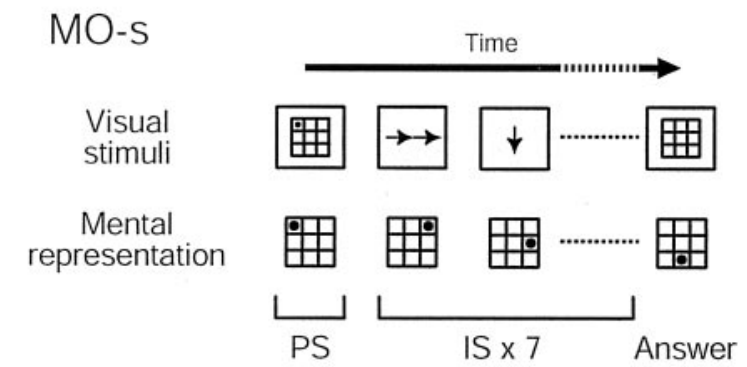

B

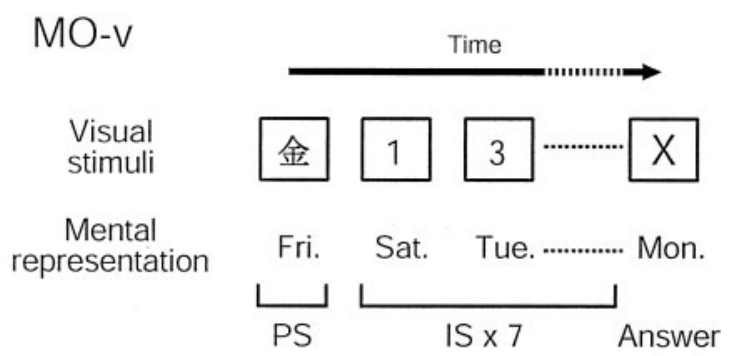

C

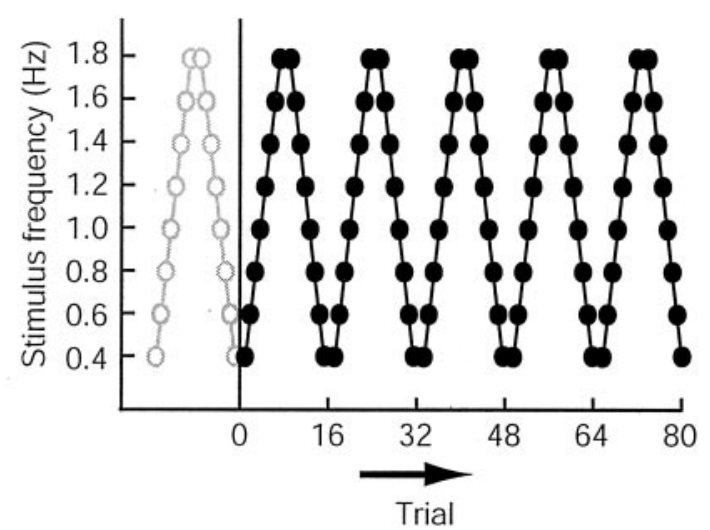

Figure 1. Experimental paradigms. A, MO-s. B, MO-v. The top row illustrates the visual stimuli used in each version of the task, and the bottom row illustrates the expected mental representations in the subjects. For both tasks, a trial started with the presentation of a prime stimulus $(P S)$, followed by the presentation of seven instruction stimuli (IS). Subjects serially manipulated mental representations according to the instruction stimulus. $C$, Schematic representations of the experimental procedure. The frequency of presentation of the instructional stimuli was constant within a trial but varied across trials. Eight different stimulus frequencies $(0.4-1.8 \mathrm{~Hz}$ in $0.2 \mathrm{~Hz}$ steps) were used for each task. Each task was broken into one practice block (open circles) and five experimental blocks ( filled circles). The stimulus frequency was serially increased in the first half of the block and then serially decreased in the second half.

\section{RESULTS}

For both the PD and control groups, the performance accuracy of the two kinds of MO tasks (MO-s and MO-v) became increasingly worse as the stimulus frequency increased (Fig. 2A,B). This finding confirms that the present method can effectively evaluate the speed of cognitive processing without involving the immediate motor response. The performance accuracy at the slowest stimulus frequency of $0.4 \mathrm{~Hz}$ was almost the same for both tasks in the patients with PD and the controls, but decreased more in the patients with PD than in the controls at greater stimulus frequencies. The difference between the two groups was most prominent at $1.0 \mathrm{~Hz}$ in both MO-s and MO-v (Fig. 2C). Statistical evaluation generally supported this interpretation. RM-ANOVA for the entire data set revealed significant main effects of group, frequency, and task and significant interactions between group and frequency, indicating that patients with PD performed with significantly lower accuracy than controls, a difference that widened with increasing stimulus frequency. Separate unpaired Student's $t$ tests revealed that the performance accuracy at the slowest stimulus frequency $(0.4 \mathrm{~Hz})$ was not different between the two groups for either task (MO-s, $p=0.30$; MO-v, $p=0.52$ ). In MO-s, the PD group exhibited relatively preserved performance at $0.6 \mathrm{~Hz}(p=0.27)$ and $0.8 \mathrm{~Hz}(p=0.17)$ but significantly $(p<$ 0.05 ) lower performance at $\geq 1.0 \mathrm{~Hz}$ stimulus frequencies, except at $1.4 \mathrm{~Hz}(p=0.07)$ (Fig. $2 A)$. In MO-v, the performance accuracy was significantly lower in patients with PD at stimulus frequencies of $\geq 0.6 \mathrm{~Hz}(p<0.05)$ (Fig. $2 B)$.

The effect of cognitive slowing on the two tasks was compared for each group (Fig. 3). MO-v was more affected than MO-s in patients with PD except at the slowest stimulus frequency. For controls, such a tendency was observed only at high stimulus frequencies. Separate RM-ANOVAs for each group demonstrated that patients with PD made more errors in MO-v $(p<$ $0.01)$, whereas controls made equal numbers of errors in both tasks $(p=0.54)$ (Table 3$)$.

In the PD group, there was a significant positive correlation between the bradyphrenia score and the bradykinesia subscale for both the MO-s $(r=0.42 ; p<0.05)$ and the MO-v $(r=0.43 ; p<$ 0.05) (Fig. 4).

To exclude a possible effect of learning or easy fatigability during the experiment, the performance accuracy for all stimulus frequencies was pooled for each different block of each task and subjected to another RM-ANOVA with a within-subject factor of "block" (from 1 to 5) and a between-subject factor of "group" (PD and controls). This analysis revealed no significant interaction between group and block in either MO-s $(p=0.10)$ or MO-v $(p=0.20)$. Therefore, the time course of task performance was not different between the two groups in either task, and the effects of easy fatigability or different learning curves of the patients with PD did not account for the poor performance in the PD group.

As a preliminary test of the sensitivity of the mental-operation tasks, an individual analysis was also performed. A confidence interval of the bradyphrenia score was calculated based on the average and two times the SD of the scores recorded from 25 controls for each task. In MO-s, five patients with PD and two controls exhibited higher bradyphrenia scores than the upper range of the confidence interval, whereas in MO-v, seven patients and one control did so.

\section{DISCUSSION}

The present results show that patients with early stage PD exhibited cognitive slowing on both the spatial and verbal versions of a mental-operation task, a difference that became more pronounced as the speed of the task increased. In both mentaloperation tasks, the task performance at the slowest stimulus frequency was the same in the patients with PD and the controls, indicating that there was no significant difference in the baseline task performance between the two groups. In contrast, the performance of patients with PD was significantly impaired com- 
A

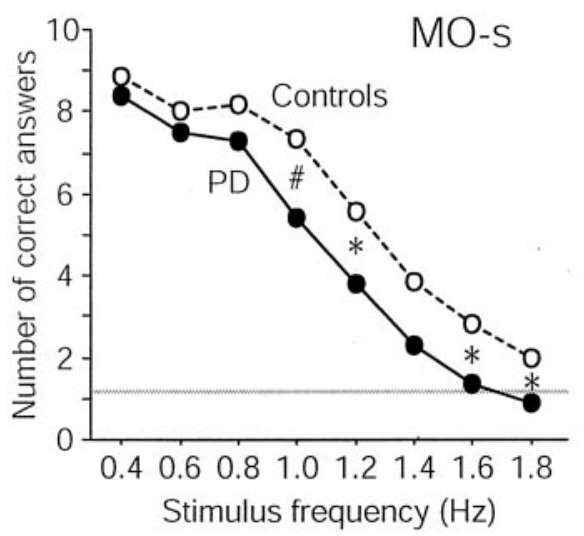

B

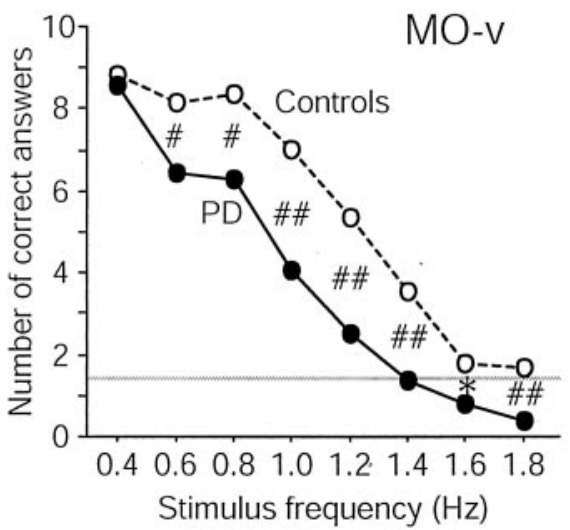

C

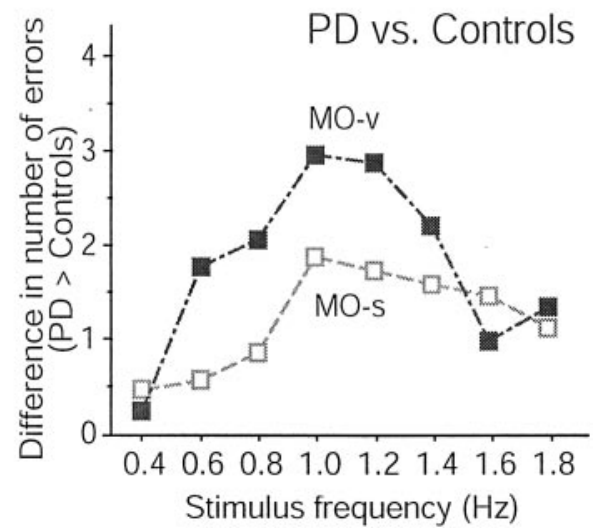

Figure 2. The number of correct answers for the MO-s $(A)$ and MO-v $(B)$ in patients with $\mathrm{PD}$ and controls. The number of correct answers for both groups decreased as the stimulus frequency increased. At stimulus frequencies of $\geq 1.4 \mathrm{~Hz}$, their performance approached chance ( gray horizontal line) or even below. $C$, Difference in the number of correct answers between patients with PD and controls at each stimulus frequency for each task. The performance deficit in patients with PD was most prominent at $1.0 \mathrm{~Hz}$ in both MO-s and MO-v. Moreover, the deficit in patients with PD was greater in MO-v compared with MO-s. ${ }^{*} p<0.05 ;{ }^{\#} p<0.01 ;{ }^{\# \#} p<0.001$.

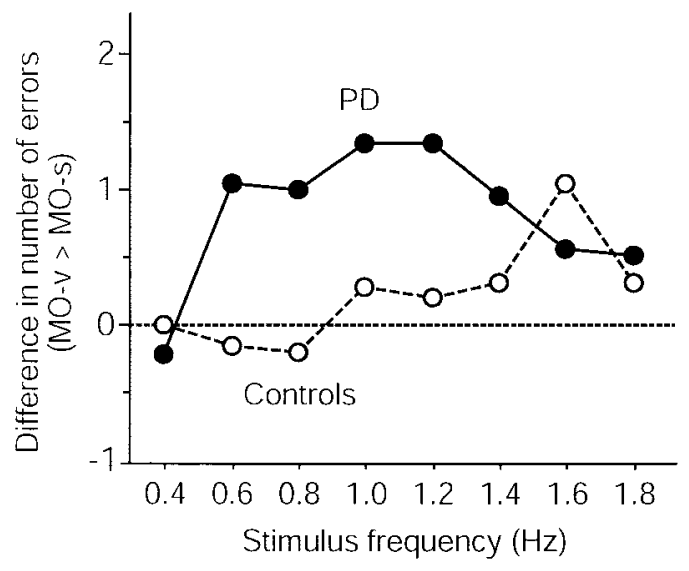

Figure 3. Difference between the number of correct answers for MO-s and MO-v at each stimulus frequency for each group. At $0.4 \mathrm{~Hz}$, the number of correct answers was the same for both tasks and for both groups. At greater speeds, the PD group exhibited a greater deficit in MO-v than MO-s. This divergence in performance level was not observed for controls. The performance at $\geq 1.4 \mathrm{~Hz}$ was difficult to evaluate because the number of correct answers was near the chance level, especially for the PD group for MO-v. The horizontal dotted line indicates equal performance level of MO-v and MO-s.

pared with controls when the cognitive task required a faster processing speed. The present findings suggest that the slowing observed in PD was not restricted to the motor domain but was observed in other domains of behavior, including cognitive mental operations.

Previous studies inferred the time required for cognitive processing from the time measured to respond using overt motor behavior (Wilson et al., 1980; Rafal et al., 1984; Pillon et al., 1989; Lafleche et al., 1990; Ransmayr et al., 1990; Poewe et al., 1991; Revonsuo et al., 1993; Cooper et al., 1994; Duncombe et al., 1994; Howard et al., 1994; Pate and Margolin, 1994; Lee et al., 1998; Berry et al., 1999). Most of those studies administered different types of reaction-time tasks comparing simple and complex cognitive processes. The time for cognitive processing was calculated by subtracting the movement time from the total reac-
Table 3. Results of statistical assessment

\begin{tabular}{llrc} 
& Effect & $F$ value & $p$ value $^{a}$ \\
\hline Combined analysis & $\mathrm{G}$ & 15.58 & $<0.001$ \\
& $\mathrm{~F}$ & 305.57 & $<0.001$ \\
& $\mathrm{~T}$ & 5.52 & $<0.05$ \\
& $\mathrm{G} \times \mathrm{F}$ & 4.30 & $<0.01$ \\
& $\mathrm{G} \times \mathrm{T}$ & 1.79 & 0.19 \\
& $\mathrm{~F} \times \mathrm{T}$ & 1.16 & 0.33 \\
& $\mathrm{G} \times \mathrm{F} \times \mathrm{T}$ & 1.40 & 0.22 \\
Separate analysis for each group & & & \\
PD & $\mathrm{F}$ & 147.00 & $<0.001$ \\
& $\mathrm{~T}$ & 10.74 & $<0.01$ \\
& $\mathrm{~F} \times \mathrm{T}$ & 1.48 & 0.22 \\
Controls & $\mathrm{F}$ & 162.74 & $<0.001$ \\
& $\mathrm{~T}$ & 0.39 & 0.54 \\
& $\mathrm{~F} \times \mathrm{T}$ & 1.03 & 0.41
\end{tabular}

G, Group (PD and controls); F, frequency (from 0.4 to $1.8 \mathrm{~Hz}$ in $0.2 \mathrm{~Hz}$ steps); T, task (MO-s and MO-v).

${ }^{a} p$ values indicate significance after Greenhouse-Geisser correction.

tion time. This procedure is based on the hypothesis that includes the assumption that cognitive and motor components of behavior occur serially, without interaction (Rabbit, 1971). However, it has been shown that patients with PD have, at least in their motor domain, difficulties in performing several behaviors sequentially or simultaneously, in addition to deficits in conducting individual behaviors separately (Schwab et al., 1954; Benecke et al., 1986, 1987; Agostino et al., 1992). These findings suggest that cognitive motor interactions are impaired in PD. In addition, a recent physiological study provides evidence that even the primary motor cortex reflects some aspects of sensory information that guide motor behavior (Georgopoulos, 2000). Moreover, reaction-time tasks usually reflect both the speed and accuracy of the performance. Speed is influenced by the level of accuracy or insistence on accuracy for lowering error rates, which usually results in increases in reaction time (Rabbit, 1971). Thus, a line of evidence indicates that cognitive processing and motor control are intimately connected in a wide range of neuronal systems and that 
MO-s at $1.0 \mathrm{~Hz}$

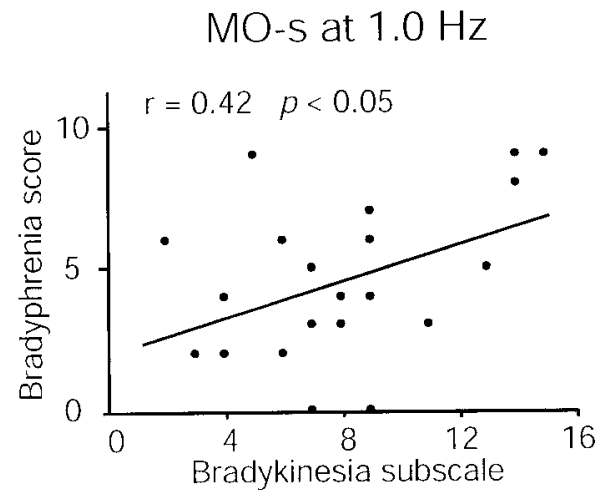

MO-v at $1.0 \mathrm{~Hz}$

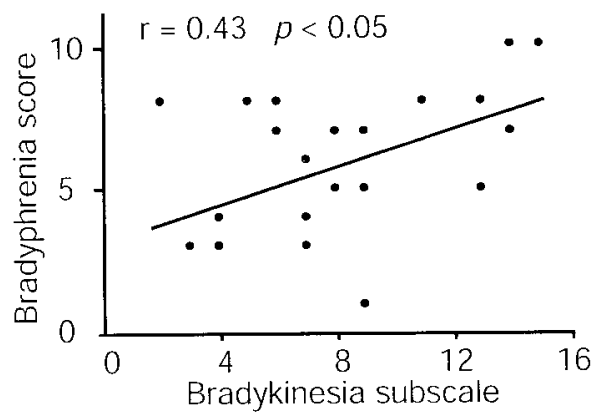

Figure 4. Correlation between the bradykinesia subscale and the bradyphrenia score for MO-s and MO-v. Correlational analysis of motor impairment and cognitive slowing in PD demonstrates a significant relationship between bradykinesia and bradyphrenia in MO-s $(r=0.42 ; p<0.05)$ and MO-v $(r=$ $0.43 ; p<0.05)$. the two processes are not easily separable (Rosenbaum et al., 2001). These characteristics make it difficult to evaluate cognitive processing speed using a reaction-time task.

An advantage of the mental-operation tasks was the ability to assess cognitive speed without involving immediate motor behavior. The cognitive speed of patients with PD was measured as a function of performance accuracy of the tasks performed at varied stimulus frequencies, instead of reaction time, so as to exclude the effect of impairment in motor preparation and execution. Thus, the slowing revealed by the mental-operation tasks was not likely attributable to bradykinesia or motor slowing, even if the task performance was related to any covert movement that may reflect motor impairments in PD.

Slowing of actions is a cardinal feature of PD. Marsden (1982) proposed that motor slowing of PD is primarily associated with motor planning impairment, but not with motor execution itself. This proposal is supported by findings that motor slowing of PD is not as pronounced in simple movements compared with complex movements, the execution of which requires motor planning to operate a number of sequential or concurrent simple movements (Schwab et al., 1954; Benecke et al., 1986, 1987; Agostino et al., 1992). Slowing of behavior not attributable to actual motor execution has also been suggested during imagery of sequential finger movements in patients with PD (Dominey et al., 1995). This study demonstrated a linear relationship between the slowing of overt motor behavior and motor imagery. Moreover, slowing in PD was demonstrated not only for imagery of personal movements but also for events in the external environment, such as motion imagery or mental rotation (Schnider et al., 1995; Lee et al., 1998). These lines of evidence are consistent with the present findings that the slowing of PD may not be restricted to the motor domain but may include the cognitive domain of behavior as well. In addition, the cognitive slowing elucidated in the present study was positively correlated with an index of the motor impairments reflecting motor slowing in PD (Vingerhoets et al., 1997). The findings suggest that cognitive slowing may share, at least in part, the same pathophysiological mechanisms as those for motor slowing.

Speed of information processing can be linked with working memory capacity, because faster rehearsal allows the maintenance of a larger amount of information in memory (Fry and Hale, 2000). Thus, the cognitive slowing demonstrated in the present study is consistent with previous findings that there are working memory impairments in PD (Owen et al., 1997). The role of the neural circuit involving the prefrontal cortex needs to be explicitly studied in the future.

Cognitive processing does not appear to be uniformly slowed in PD, as observed also for motor responses (Majsak et al., 1998).
The present findings demonstrate that the degree of performance decline associated with an increase in stimulus frequency in PD was greater on MO-v compared with MO-s. This deficit may be attributed to several factors, one of which may be the requirement of sequential processing. In MO-v, the subjects had to form mental representations of the days of the week in sequential order. The sequential processing is thought to be essential to the use of language and may be generally involved in verbal mental operations (Burgess and Hitch, 1999). Previous studies have shown that there is remarkable slowing of sequential motor behavior in patients with PD (Benecke et al., 1987; Agostino et al., 1992). Alternatively, slower performance on MO-v may stem from greater demand for internally guided behavior in MO-v compared with MO-s. Presumably, cognitive processing during MO-s was executed each time in response to external visual cues. In contrast, in addition to information processing directly guided by external cues, MO-v required the subject to advance the day of the week using an internally guided mechanism, particularly in response to the presentation of the number 2 or 3 . It has been suggested that the internal cueing mechanism underlying selfinitiated movement is impaired in PD and that patients rely on external cues to compensate for the motor impairments (Georgiou et al., 1993). Therefore, we suggest that such an internal cueing mechanism may be used not only for motor control but also for guiding cognitive processing (Brown and Marsden, 1988). Another possible explanation for the present results may be a deficit of PD in switching attention resources from one to another as required in a task (Cools et al., 1984; Brown and Marsden, 1991). In MO-s, arrows instructed an operation on a location of the marker, both of which are concrete spatial representations. In contrast, MO-v used a number to guide an operation on the day of the week. Switching attention resources between two different symbolic representations in MO-v may cause a greater increase in processing time.

Using $\mathrm{H}_{2}{ }^{15} \mathrm{O}$ PET and primarily the same tasks as those for the present study, we have shown that the striatum and the medial premotor cortex exhibited greater activity during MO-v compared with MO-s in healthy subjects, whereas the lateral premotor cortex exhibited greater activity during MO-s than during MO-v (Honda et al., 1998). The striatum and medial premotor cortex have been implicated in the control of sequential motor behavior rather than in the execution of individual movements (Boecker et al., 1998). In addition, the striatum and medial premotor cortex appear to be essential for internally cued movements (Rao et al., 1997). A neurocognitive model of language suggests that the rule-governed combination of words depends on procedural memory that appears to involve the striatum and medial premotor 
cortex (Miyachi et al., 1997; Nakamura et al., 1998; Ullman, 2001). Moreover, previous neuroimaging studies in patients with PD suggest that there are deficits in the striatum and medial premotor cortex during internally generated movements (Playford et al., 1992; Hanakawa et al., 1999b) and relatively preserved or even hyperactive function in the lateral premotor cortex, at least in motor tasks (Samuel et al., 1997; Catalan et al., 1999; Hanakawa et al., 1999a). This line of evidence raises the possibility that the difference in the degree of cognitive slowing according to the nature of the tasks may be attributed to the dysfunction of the striatum or medial premotor cortex, or both, in PD.

In summary, the present results demonstrate cognitive slowing in patients with PD by using mental-operation tasks that assess cognitive processing speed without a motor component. The present results also suggest that the level of cognitive slowing in PD was correlated with the level of motor slowing. Together with our PET results obtained during the performance of the same tasks, these results indicate that dysfunction of the striatum or medial premotor cortex may be responsible not only for motor deficits but also for cognitive disturbance in PD. The present results support the concept that motor and cognitive slowing have a common framework in PD.

\section{REFERENCES}

Agostino R, Berardelli A, Formica A, Accornero N, Manfredi M (1992) Sequential arm movements in patients with Parkinson's disease, Huntington's disease and dystonia. Brain 115:1481-1495.

Benecke R, Rothwell JC, Dick JP, Day BL, Marsden CD (1986) Performance of simultaneous movements in patients with Parkinson's disease. Brain 109:739-757.

Benecke R, Rothwell JC, Dick JP, Day BL, Marsden CD (1987) Disturbance of sequential movements in patients with Parkinson's disease. Brain 110:361-379.

Berry EL, Nicolson RI, Foster JK, Behrmann M, Sagar HJ (1999) Slowing of reaction time in Parkinson's disease: the involvement of the frontal lobes. Neuropsychologia 37:787-795.

Boecker H, Dagher A, Ceballos-Baumann AO, Passingham RE, Samuel M, Friston KJ, Poline J, Dettmers C, Conrad B, Brooks DJ (1998) Role of the human rostral supplementary motor area and the basal ganglia in motor sequence control: investigations with H2 $15 \mathrm{O}$ PET. J Neurophysiol 79:1070-1080.

Brown RG, Marsden CD (1988) Internal versus external cues and the control of attention in Parkinson's disease. Brain 111:323-345.

Brown RG, Marsden CD (1991) Dual task performance and processing resources in normal subjects and patients with Parkinson's disease. Brain 114:215-231.

Burgess N, Hitch GJ (1999) Memory for serial order: a network model of the phonological loop and its timing. Psychol Rev 106:551-581.

Catalan MJ, Ishii K, Honda M, Samii A, Hallett M (1999) A PET study of sequential finger movements of varying length in patients with Parkinson's disease. Brain 122:483-495.

Cools AR, van den Bercken JH, Horstink MW, van Spaendonck KP, Berger HJ (1984) Cognitive and motor shifting aptitude disorder in Parkinson's disease. J Neurol Neurosurg Psychiatry 47:443-453.

Cooper JA, Sagar HJ, Tidswell P, Jordan N (1994) Slowed central processing in simple and go/no-go reaction time tasks in Parkinson's disease. Brain 117:517-529.

Dominey P, Decety J, Broussolle E, Chazot G, Jeannerod M (1995) Motor imagery of a lateralized sequential task is asymmetrically slowed in hemi-Parkinson's patients. Neuropsychologia 33:727-741.

Duncombe ME, Bradshaw JL, Iansek R, Phillips JG (1994) Parkinsonian patients without dementia or depression do not suffer from bradyphrenia as indexed by performance in mental rotation tasks with and without advance information. Neuropsychologia 32:1383-1396.

Fry AF, Hale S (2000) Relationships among processing speed, working memory, and fluid intelligence in children. Biol Psychol 54:1-34.

Georgiou N, Iansek R, Bradshaw JL, Phillips JG, Mattingley JB, Bradshaw JA (1993) An evaluation of the role of internal cues in the pathogenesis of parkinsonian hypokinesia. Brain 116:1575-1587.

Georgopoulos AP (2000) Neural aspects of cognitive motor control. Curr Opin Neurobiol 10:238-241.

Hanakawa T, Fukuyama H, Katsumi Y, Honda M, Shibasaki H (1999a) Enhanced lateral premotor activity during paradoxical gait in Parkinson's disease. Ann Neurol 45:329-336.

Hanakawa T, Katsumi Y, Fukuyama H, Honda M, Hayashi T, Kimura J, Shibasaki H (1999b) Mechanisms underlying gait disturbance in Par- kinson's disease: a single photon emission computed tomography study. Brain 122:1271-1282.

Honda M, Hanakawa T, Okada T, Sawamoto N, Sadato N, Nakamura S, Waki A, Yonekura Y, Fukuyama H, Shibasaki H (1998) Lateral and medial area 6 plays a role in cognitive operations nonspecific to motor controls in humans: a combined PET and fMRI study. Soc Neurosci Abstr 24:1262.

Howard LA, Binks MG, Moore AP, Playfer JR (1994) How convincing is the evidence for cognitive slowing in Parkinson's disease? Cortex 30:431-443.

Lafleche GC, Stuss DJ, Nelson RG, Picton TW (1990) Memory scanning and structured learning in Alzheimer's dementia and Parkinson's disease. Can J Aging 9:120-134.

Lee AC, Harris JP, Calvert JE (1998) Impairments of mental rotation in Parkinson's disease. Neuropsychologia 36:109-114.

Majsak MJ, Kaminski T, Gentile AM, Flanagan JR (1998) The reaching movements of patients with Parkinson's disease under self-determined maximal speed and visually cued conditions. Brain 121:755-766.

Marsden CD (1982) The mysterious motor function of the basal ganglia: the Robert Wartenberg Lecture. Neurology 32:514-539.

Middleton FA, Strick PL (1994) Anatomical evidence for cerebellar and basal ganglia involvement in higher cognitive function. Science 266:458-461.

Miyachi S, Hikosaka O, Miyashita K, Karadi Z, Rand MK (1997) Differential roles of monkey striatum in learning of sequential hand movement. Exp Brain Res 115:1-5.

Nakamura K, Sakai K, Hikosaka O (1998) Neuronal activity in medial frontal cortex during learning of sequential procedures. J Neurophysiol 80:2671-2687.

Naville F (1922) Etudes sur les complications et les sequelles mentales de l'encephalite epidemique: la bradyphrenie. Encephale 17:369-375.

Owen AM, Iddon JL, Hodges JR, Summers BA, Robbins TW (1997) Spatial and non-spatial working memory at different stages of Parkinson's disease. Neuropsychologia 35:519-532.

Pate DS, Margolin DI (1994) Cognitive slowing in Parkinson's and Alzheimer's patients: distinguishing bradyphrenia from dementia. Neurology 44:669-674.

Pillon B, Dubois B, Bonnet AM, Esteguy M, Guimaraes J, Vigouret JM, Lhermitte F, Agid Y (1989) Cognitive slowing in Parkinson's disease fails to respond to levodopa treatment: the 15-objects test. Neurology 39:762-768

Playford ED, Jenkins IH, Passingham RE, Nutt J, Frackowiak RS, Brooks DJ (1992) Impaired mesial frontal and putamen activation in Parkinson's disease: a positron emission tomography study. Ann Neurol 32:151-161.

Poewe W, Berger W, Benke T, Schelosky L (1991) High-speed memory scanning in Parkinson's disease: adverse effects of levodopa. Ann Neurol 29:670-673.

Rabbit PM (1971) Times for the analysis of stimuli and for the selection of responses. Br Med Bull 27:259-265.

Rafal RD, Posner MI, Walker JA, Friedrich FJ (1984) Cognition and the basal ganglia: separating mental and motor components of performance in Parkinson's disease. Brain 107:1083-1094.

Ransmayr G, Bitschnau W, Schmidhuber-Eiler B, Berger W, Karamat E, Poewe W, Kemmler GW (1990) Slowing of high-speed memory scanning in Parkinson's disease is related to the severity of parkinsonian motor symptoms. J Neural Transm Park Dis Dement Sect 2:265-275.

Rao SM, Harrington DL, Haaland KY, Bobholz JA, Cox RW, Binder JR (1997) Distributed neural systems underlying the timing of movements. J Neurosci 17:5528-5535.

Revonsuo A, Portin R, Koivikko L, Rinne JO, Rinne UK (1993) Slowing of information processing in Parkinson's disease. Brain Cogn 21:87-110.

Rogers D (1986) Bradyphrenia in parkinsonism: a historical review. Psychol Med 16:257-265.

Rosenbaum DA, Carlson RA, Gilmore RO (2001) Acquisition of intellectual and perceptual-motor skills. Annu Rev Psychol 52:453-470.

Samuel M, Ceballos-Baumann AO, Blin J, Uema T, Boecker H, Passingham RE, Brooks DJ (1997) Evidence for lateral premotor and parietal overactivity in Parkinson's disease during sequential and bimanual movements: a PET study. Brain 120:963-976.

Schnider A, Gutbrod K, Hess CW (1995) Motion imagery in Parkinson's disease. Brain 118:485-493.

Schwab RS, Chafetz ME, Walker S (1954) Control of two simultaneous voluntary motor acts in normals and in parkinsonism. Arch Neurol Psychiatry 72:591-598.

Tiffin J, Asher EJ (1948) Purdue pegboard: norms and studies of reliability and validity. J Appl Psychol 32:234-247.

Ullman MT (2001) A neurocognitive perspective on language: the declarative/procedural model. Nat Rev Neurosci 2:717-726.

Vingerhoets FJ, Schulzer M, Calne DB, Snow BJ (1997) Which clinical sign of Parkinson's disease best reflects the nigrostriatal lesion? Ann Neurol 41:58-64.

Wilson RS, Kaszniak AW, Klawans HL, Garron DC (1980) High speed memory scanning in parkinsonism. Cortex 16:67-72. 